\title{
EOSINOPHILIC STATE, ASTHMA, RADICULITIS, MYOSITIS, AND LARGE VESSEL OBSTRUCTION: ATYPICAL EOSINOPHILIC GRANULOMATOSIS WITH POLYANGIITIS?
}

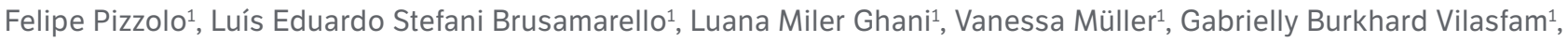
Ana Luiza Leal de Mello ${ }^{1}$, Diogo Zamprogna de Barcellos ${ }^{1}$, Eduarda Garcia Colao ${ }^{1}$, Eduarda Herscovitz Jaeger ${ }^{1}$, Laura Gazal Passos $^{1}$, Letícia Mariel König de Souza ${ }^{1}$, Erika Biegelmeyer ${ }^{1}$, Julia Boechat Farani ${ }^{1}$, Viviane Elisa Timm ${ }^{1}$, Samanta Gerhardt ${ }^{1}$, Evelise Berlet ${ }^{1}$, Lucas Immich Gonçalves ${ }^{1}$, Rafael Saidelles ${ }^{1}$, Jefferson Becker ${ }^{1}$, Henrique Luiz Staub ${ }^{1, \star}$

1.Pontifícia Universidade Católica, Porto Alegre (RS), Brazil.

${ }^{\star}$ Corresponding author: henriquestaub@terra.com.br

\section{BACKGROUND}

Eosinophilic states encompass a variety of systemic disorders. Eosinophilic granulomatosis with polyangiitis (EGPA) is characterized by atopic features, peripheral eosinophilia and small-vessel vasculitis. We herein present an interesting case of a hypereosinophilic state reminding EGPA whereby the neurological and vascular manifestations were rather intriguing.

\section{CASE REPORT}

The patient, a 60-year-old female, presented a refractory asthma since childhood. Intermittent rhinosinusitis and polyarthralgia were referred in the last months. The patient was recently admitted to the hospital due to adynamia, loss of strength in right leg and walking difficulty. Physical examination confirmed a right foot drop. Electroneuromyography (ENMG) revealed a L5 radiculopathy. Lumbar spine magnetic resonance image demonstrated inflammatory enhancement of cauda equina roots and paravertebral musculature. The chest tomography showed a diffuse bronchopathy. A marked eosinophilia was seen in blood cell count (2,391 cells $\left./ \mathrm{mm}^{3}\right)$, and inflammatory markers were elevated. Levels of creatine kinase (CK) were initially very high (5,157 units). Rheumatoid factor testing was strongly positive (138 units), but antinuclear factors were absent. A search for ANCA resulted negative. The protein electrophoresis demonstrated a polyclonal hypergammaglobulinemia. Total IgE levels were normal. Muscle biopsy demonstrated atrophic myofibers, fundamentally. In the sequence, she developed a sudden abdominal discomfort, and the angiotomography confirmed an aortoiliac stenosis requiring by-pass. After the procedure, a digit necrosis established in the right foot, probably due to embolization. A foot drop was observed in the other leg, and a new ENMG showed a sensory-motor neuropathy. Creatine kinase levels normalized, and eosinophilia disappeared. Finally, she developed an uncomplicated severe acute respiratory syndrome coronavirus 2 (SARS-CoV-2) pneumonia. At the moment, the patient is on $50 \mathrm{mg}$ of prednisone and azathioprine.

\section{CONCLUSION}

This is a thought-provoking case of an eosinophilic state with some EGPA features, such as asthma, rhinosinusitis, arthralgia and hypergammaglobulinemia. Nonetheless, a number of other findings (inflammatory cauda equina radiculitis, myositis, large vessel obstruction, ANCA negativity) were atypical or unexpected for EGPA, making the case a diagnostic challenge. Whether or not the SARS-CoV-2 exposure contributed to the late vascular features, it is also debatable. 